\title{
Perspective \\ Potential of Time-Resolved Serial Femtosecond Crystallography Using High Repetition Rate XFEL Sources
}

\author{
Raphaël de Wijn ${ }^{1, *,+}$, Diogo V. M. Melo ${ }^{1,+} \mathbb{D}$, Faisal H. M. Koua ${ }^{1,+}(\mathbb{D})$ and Adrian P. Mancuso ${ }^{1,2, *(\mathbb{D})}$ \\ 1 European XFEL GmbH, Holzkoppel 4, D-22869 Schenefeld, Germany; diogo.melo@xfel.eu (D.V.M.M.); \\ faisal.koua@xfel.eu (F.H.M.K.) \\ 2 Department of Chemistry and Physics, La Trobe Institute for Molecular Science, La Trobe University, \\ Melbourne, VIC 3086, Australia \\ * Correspondence: raphael.de.wijn@xfel.eu (R.d.W.); adrian.mancuso@xfel.eu (A.P.M.) \\ + These authors contributed equally to this work.
}

check for updates

Citation: de Wijn, R.; Melo, D.V.M.; Koua, F.H.M.; Mancuso, A.P.

Potential of Time-Resolved Serial

Femtosecond Crystallography Using High Repetition Rate XFEL Sources. Appl. Sci. 2022, 12, 2551. https:// doi.org/10.3390/app12052551

Academic Editor: Emiliano Principi

Received: 31 January 2022

Accepted: 25 February 2022

Published: 28 February 2022

Publisher's Note: MDPI stays neutral with regard to jurisdictional claims in published maps and institutional affiliations.

Copyright: (c) 2022 by the authors. Licensee MDPI, Basel, Switzerland. This article is an open access article distributed under the terms and conditions of the Creative Commons Attribution (CC BY) license (https:/ / creativecommons.org/licenses/by/ $4.0 /)$.

\begin{abstract}
This perspective review describes emerging techniques and future opportunities for timeresolved serial femtosecond crystallography (TR-SFX) experiments using high repetition rate XFEL sources. High repetition rate sources are becoming more available with the European XFEL in operation and the recently upgraded LCLS-II will be available in the near future. One efficient use of these facilities for TR-SFX relies on pump-probe experiments using a laser to trigger a reaction of light-responsive proteins or mix-and-inject experiments for light-unresponsive proteins. With the view to widen the application of TR-SFX, the promising field of photocaged compounds is under development, which allows the very fast laser triggering of reactions that is no longer limited to naturally light-responsive samples. In addition to reaction triggering, a key concern when performing an SFX experiment is efficient sample usage, which is a main focus of new high repetition ratecompatible sample delivery methods.
\end{abstract}

Keywords: megahertz repetition rate; XFEL; SPB/SFX scientific instrument; serial femtosecond crystallography; time-resolved serial femtosecond crystallography; sample delivery; liquid jet; pump-probe; photocage

\section{Introduction}

The study of biomacromolecular reactions is relevant for biology and medicine, though it is also highly challenging, especially when it needs to be performed in real time and at near-atomic resolution. Since the end of the 1950s and with the first two protein structures, myoglobin [1] and hemoglobin [2], having been solved, X-ray crystallography has been the most successful method to elucidate near-atomic resolution macromolecular structures.

Serial femtosecond crystallography (SFX) has recently evolved from traditional X-ray crystallography with the emergence of X-ray free electron lasers and their very short and brilliant X-ray pulses [3-5]. Due to this brilliance, macromolecular crystals are destroyed immediately after (or even during) [6] diffraction and thus, need to be constantly replenished using a dedicated sample delivery method. Based on this "diffraction before destruction" principle [7], short X-ray pulses allow effectively radiation damage-free studies [8], making it very relevant for radiation-sensitive biological samples. As a consequence, the protection of the crystals by cryo-cooling is no longer needed, thereby avoiding the eternal discussion in the structural biology field regarding the non-physiological temperature at which structures are determined. SFX in XFELs also enables the study of small crystals down to nanometer size [9], which is difficult in synchrotron beamlines, and avoids the inherent difficulty of growing large and well-structured crystals, especially for highly sensitive and complex samples, such as membrane proteins. As well as this crystal size requirement, crystals still need to present a good quality, i.e., good diffraction power to near-atomic resolution to allow mechanistic insight. Due to the constant supply of samples at the X-ray 
interaction point, in all but exceptional circumstances, only one diffraction pattern is ever collected per crystal, meaning that a dataset needs the exposure of and data collection from several thousands to hundreds of thousands of crystals to be completed [3]. This number of required samples is not trivial to deliver, nor is such a large amount of data necessarily easy to collect.

The European X-ray Free Electron Laser (EuXFEL) in Schenefeld, Germany, is the only running source of megahertz repetition rate XFEL pulses currently available to users. With the Single Particles, Clusters and Biomolecules and Serial Femtosecond Crystallography (SPB/SFX) instrument of the EuXFEL that is equipped for SFX experiments, the typical X-ray pattern consists of X-ray pulse trains delivered at $10 \mathrm{~Hz}$ with each train comprising up to 352 pulses separated by $880 \mathrm{~ns}$, leading to an intra-train repetition rate of $1.1 \mathrm{MHz}[10,11]$. In its current configuration, a 1 Megapixel Adaptive Gain Integrating Pixel Detector (AGIPD 1M) is able to collect data at megahertz repetition rate, but it only operates in a vacuum environment [11,12]. See [13] and references therein for a detailed description of a number of SFX experiments performed using the SPB/SFX instrument. The most important advantage of SFX at high repetition rate facilities is the ability to collect data at a much faster rate than other XFELs: at least 30 times faster for the EuXFEL $[5,9,14,15]$. In practice, this means that $10 \mathrm{~min}$ of data collection at the SPB/SFX instrument produces the same amount of data as more than $5 \mathrm{~h}$ of data collection at any other currently available XFEL running at a low repetition rate. However, megahertz repetition rate and sample injection in vacuum come with limitations that need to be overcome, such as the sample needing to be replaced at the same rate and without freezing. Due to these constraints, the most commonly used sample delivery method is within a low viscosity liquid jet $[16,17]$. Currently, it is the only method available to replenish the sample fast enough to bring a fresh crystal to the interaction point before the subsequent $X$-ray pulse is delivered while still remaining flexible enough to allow time-resolved studies in the form of mix-and-inject and pump-probe experiments [18-20]. Alternative methods are being tested as to whether they can achieve better results than the standard liquid jets while remaining compatible with the previously mentioned constraints.

Time-resolved serial femtosecond crystallography (TR-SFX) is defined by the study of biomacromolecular reactions observed with time resolutions as rapid as femtosecond-scale using SFX [15,21,22]. Since the first published TR-SFX experiment in 2012 at LCLS [23], many studies have been reported [24-31], including those conducted at the European XFEL that made use of its ultra-fast repetition rate $[18,32,33]$. As time-resolved studies require much more data to be collected in comparison to regular SFX ( $N$ times more, where $N$ is the number of desired time points to obtain reliable difference electron density maps), the megahertz repetition rate represents a significant advantage in data collection time [14]. For time-resolved experiments, a method to trigger the enzymatic reaction is needed and the two most common methods are laser triggering (pump-probe) and mixing (mix-andinject) $[19,20,32]$. Furthermore, a very promising field under development is the use of caged compounds, allowing the very fast laser triggering of a reaction with a much wider spectrum of applications [34-36].

This perspective review aims to describe recent work performed in fields related to TR-SFX at high repetition rate XFELs, including sample delivery methods, mix-andinject and pump-probe experiments, as well as the development of caged compounds. After discussing their advantages and limitations, we highlight possible further developments to make TR-SFX more broadly available to user groups and applicable to more scientific questions.

\section{Sample Delivery Methods}

\subsection{Actual Standard Sample Delivery Methods}

A major concern when performing SFX, especially TR-SFX as a higher number of datasets are required, is the large sample number that is needed. Based on our experience, for a static dataset, the average number can vary between a few hundreds of microliters to 
milliliters of crystal suspension with around 25\% $(v / v)$ crystals, based on hit rate, sample quality and injection method. This is especially relevant for megahertz repetition rate X-ray facilities operating with bunch patterns, such as the EuXFEL, and is an important challenge for sample production laboratories, which limits the scope of sample availability. At the moment, the AGIPD 1M is one of the few detectors able to operate at a megahertz rate with the unique pulse pattern of the EuXFEL [11,12]. Sample injection in a vacuum at such repetition rates is a difficult task and is currently limited to a few methods and devices that are able to prevent freezing and replenish the sample fast enough. The most commonly used injection method at the SPB/SFX instrument that is compatible with the abovementioned constraints is liquid jets, based either on a gas dynamic virtual nozzle (GDVN) (Figure 1a) [37] or a double flow focusing nozzle (DFFN) [38] (Figure 1b). Both of these types of nozzles contain an internal and an external capillary, which allow the flow of the sample and helium gas to accelerate and focus the sample stream, respectively. DFFNs contain an additional capillary to deliver a focusing solution, usually ethanol [38]. GDVNs are well-characterized devices and have been used since 2008 [37,39-41]. They were first made of glass capillaries and had a manual method, meaning that each nozzle was different, their preparation time consuming and they required specific expertise. Recently, the increasing availability of 3D printing methods and 2-photon polymerization techniques has improved the production of GDVNs with high quality and high reproducibility $[16,42,43]$. However, even with such a novel nozzle production method, the standard liquid jets still have limitations regarding: their sensitivity to clogging issues, owing to the sample viscosity or crystal preparation; their high sample consumption, as the sample needs to be continuously flowing even when no X-rays are present; and high flow rates are needed for stable jetting. In standard liquid jet injection, 300 times more sample is injected than is actually probed by the X-rays. DFFNs, with the use of a focusing liquid, address these limitations, as they allow the use of higher viscosity samples and reduce the minimal sample flow rate for stable injection by a factor of $4[16,42]$. Notwithstanding these improvements, they still do not solve the problem of wasted samples (amounting to more than $99 \%$ ) and generate additional background due to the sheathing liquid. Very recently, Vakilii et al. presented extensive details about all current sample delivery methods available at the EuXFEL, including those used at the SPB/SFX instrument [16]. In the next section, we highlight the current developments that are underway to cope with such limitations.
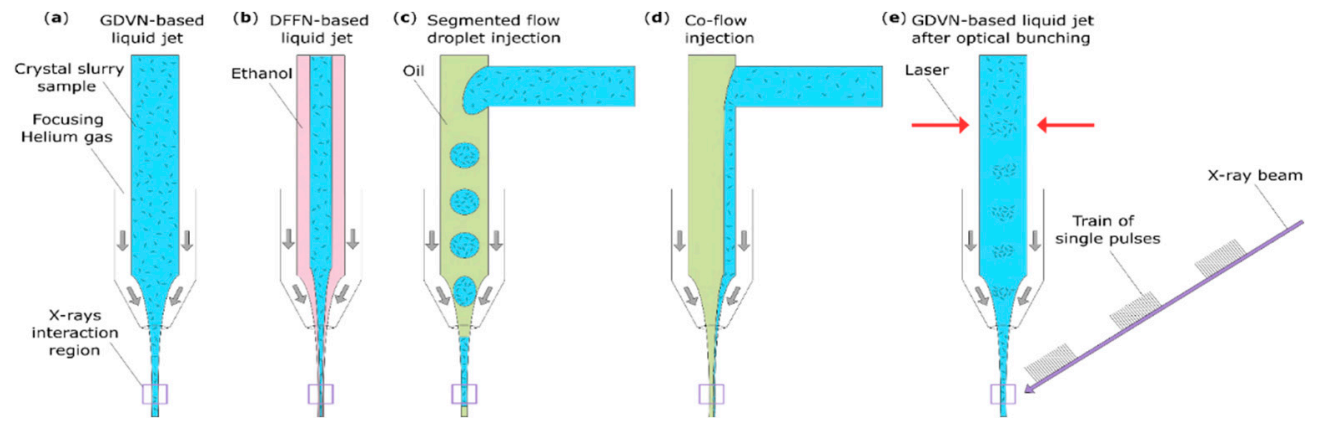

Figure 1. Schematic representations of standard $(\mathbf{a}, \mathbf{b})$ and innovative (c-e) injection delivery methods that are compatible with megahertz operation at the EuXFEL: (a) a GDVN-based liquid jet; (b) a DFFN-based liquid jet [38]; (c) a co-flow injection [44]; (d) a segmented flow droplet injection [45]; (e) a GDVN-based liquid jet with optical bunching [46].

\subsection{Innovative Sample Delivery Methods}

Efficient sample consumption is one of the key elements of emerging and innovative injection delivery systems. The methods that allow for a lower sample consumption, such as tape drive, some drop-on-demand injectors $[47,48]$ and fixed target or viscous media injecting devices [49,50], are incompatible or very difficult to adapt to megahertz rate operation in a vacuum. Fixed target holders that are compatible with operation at high 
repetition rates are possible and are currently under development, but they are still far from ready and available as standard.

Future methods also need to be compatible with sub-micrometer crystals, i.e., they must exhibit a low solvent background. Most of these rely on the synchronization of the sample delivery with the arrival of the X-ray pulse either by bunching the crystals (optical bunching [46]), injecting an alternative liquid during the dark time (segmented flow droplet injection [45]) or aiming to reduce the sample amount needed for a stable jet [38,44] (see Figure 1).

The segmented flow droplet injection (Figure 1c) is one approach that can be used to reduce sample wastage in SFX experiments and has already been tested at the European XFEL [45]. It aims to generate sub-nanoliter crystal suspension droplets embedded in an immiscible oil, allowing for injection with a traditional GDVN. Even without the synchronization of the droplet with the $\mathrm{X}$-ray pulse train, this approach has already shown a sample reduction of $60 \%$. Theoretically, with perfect synchronization, sample consumption could be reduced by $99 \%$, as no sample would be injected within the period where X-rays are not delivered (so called "dark" or "dead" time). Recent work at the EuXFEL has demonstrated the use of a variation of the segmented flow method in the form of a hybrid microfluidic 3D-printed co-flow device coupled with a GDVN [44] (Figure 1d). By injecting the sample immediately before injection through a side channel into the main capillary, which contains an immiscible oil, and tuning the flow rates, they have managed to deliver a stable liquid jet and reduce clogging effects. With this method, sample consumption could theoretically be reduced by $95 \%$, whereas in this study sample, consumption was reduced by $75 \%$. This reduction is achieved by lowering the sample flow rate that is needed to establish the liquid jet, but sample delivery is still continuous and thus, wasted during the "dark" time.

The optical bunching method (Figure 1e) relies on the use of a laser setup to create an optical trap in the capillary to periodically trap and release concentrated crystals [46]. In the first description of this method, Awel et al. demonstrated a 30-fold local sample increase in the concentration into $10 \mathrm{~Hz}$ bunches using polystyrene particles. If this technique is proven to be usable with biomacromolecular crystals during sample delivery with a GDVN, it could theoretically allow for a 20-fold reduction in sample concentration without impacting the hit rate compared to the regular liquid jets with a GDVN and thus, lead to a $95 \%$ reduction in the sample consumption.

The development of these innovative sample delivery methods focuses on preserving the advantages of liquid jets, i.e., being able to deliver samples fast enough for the high repetition rate XFELs without freezing the sample in a vacuum as well as its flexibility, while addressing its major limitation of the sample wastage. This development raises other questions and difficulties. For instance, can the crystalline sample be altered by its interaction with ethanol or with the laser providing the optical trap for DFFNs and the optical bunching method, respectively? How can we perfectly synchronize the sample droplets with the arriving X-ray pulses in the segmented flow method? Is the number of diffraction patterns collected from milligrams of protein equal in all of these delivery methods? These are open research questions presently under investigation within the SFX community.

\section{Time-Resolved Serial Femtosecond Crystallography}

The advancement of the hard X-ray free electron lasers technology with its unique ultrashort and highly brilliant pulses at various XFEL sources has revolutionized the field of structural biology by bringing about a significant development in time-resolved crystallography (TRX), thereby leading to the emergence of the TR-SFX $[4,5,9,14,15,20]$. The TR-SFX made possible the direct visualization of biomacromolecules, e.g., proteins and nucleic acids, in action inside the crystals and under their near-physiological conditions with high spatial and temporal resolutions $[9,14,15]$. Almost all biochemical reactions are controlled by the actions of series of proteins or protein-nucleic acids that undergo essential conformational changes as a function of time following the triggering of the reaction by a 
specific factor that enables a specific set of reactions to subsequently occur $[25,26,51-53]$. The trigger of a biochemical reaction can be any chemical or physical factor that elicits the biomacromolecule to react and realize the specific biological function $[14,15,51]$. The TR-SFX enables the probing of these conformational changes at different delay time points and thus, significantly aids the understanding of the mechanisms of these processes at near-atomic levels [15]. Based on the triggering system, the TR-SFX can be divided into two major categories: (i) the pump-probe TR-SFX, which uses light excitation (physical factor) to trigger the biochemical reaction of the biomacromolecule [23], and (ii) mix-andinject TR-SFX, which uses a chemical trigger or substrate (chemical factor) to trigger the reaction [26]. Using the SPB/SFX instrument and high-repetition rate X-rays, the standard pulse duration available for users is nominally $25 \mathrm{fs}$ (potentially down to less than $10 \mathrm{fs}$ upon request) with a jitter of $\sim 12$ fs [54], corresponding to the shortest time delay available for time-resolved studies with laser excitation. There is no limitation on the longest time delay, as the trigger for a pump-probe or the "start" of a mixing experiment can be an almost arbitrary amount of time prior to the X-ray's interaction with the sample. In the following subsections, we highlight some of the recent advancements in the pump-probe TR-SFX approach with particular a focus on experiments that took place using the SPB/SFX instrument of the European XFEL facility, as well as on mix-and-inject TR-SFX and the development and use of cage compounds.

\subsection{TR-SFX with Light-Responsive Biomacromolecules}

In the last decade and with the advent of hard X-ray free electron laser technology, the pump-probe TR-SFX approach has witnessed a significant development that has led to a spectacular advancement in the structural and dynamical studies of some highly challenging biological systems, i.e., light-responsive biological systems, such as photosynthetic proteins, phytochromes and microbial rhodopsins [24,25,28,31,55-57]. In 2012, Aquila et al. conducted the first TR-SFX experiments using the CFEL-ASG multi-purpose instrument at the Linac Coherent Light Source (LCLS) on the photosystem I-ferredoxin complex, where they used an Nd:YLF optical pump laser system to illuminate the complex and then probed the photo-induced structural changes that occurred within 5 to $10 \mu$ s delay times using the $X$-ray free electron laser [23]. This pioneering pump-probe TR-SFX experiment, accompanied by the advances in the application of pump lasers and microcrystal delivery methods, has paved the way to rapid development within the study of the structure and dynamics of light-sensitive biomacromolecules [18,24,26-28,58]. In the pump-probe TR-SFX, an optical 'pump' laser is used to trigger the biochemical reaction in crystallo by illuminating the microcrystals with defined optical laser pulses at a defined wavelength and energy in a controlled region of the jet and/or nozzle before probing the reactions in crystallo with X-ray FEL pulses 'probe' at different delay times to allow observing the reaction of the biomacromolecule in real time (Figure 2) $[18,23,24]$.

There are several parameters that need to be considered and carefully tuned for a successful pump-probe TR-SFX experiment. The most obvious are the timing synchronization between the optical laser "pump" and the FEL "probe", the optical laser power, the materials used for the fabrication of the injection device and the optical density or the thickness and size of the microcrystals $[18,23,54,59-62]$. The synchronization of the X-ray FEL and optical laser pulses is critical for the accurate measurement of delay time points for experiments targeting the dynamics of timescales from fs to a few ps and hence, the data interpretation of the pump-probe SFX experiments, particularly for the instruments that run at a megahertz repetition rate $[11,54,60]$. With the SPB/SFX instrument at the European XFEL, significant progress has been made by improving the temporal resolution and decreasing the shot-to-shot timing fluctuations to the lowest ever megahertz rate timing jitter of only $24.0 \mathrm{fs}$ RMS, with an uncertainty of $12.4 \mathrm{fs}$ [54] and the ongoing development to further reduce the jitter. In 2020, Pandey et al. used micron-sized photoactive yellow protein (PYP) crystals to explore the initial isomerization events of the $p$-coumarin chromophore as well as the early stage intermediates of the photocycle, as early as 10-100 ps 
delay time points using the SPB/SFX instrument operating at $\mathrm{MHz}$ repetition rate [18]. The TR-SFX datasets showed excellent difference electron densities and allowed the authors to observe the clear torsional angle increase in the $p$-coumarin skeleton along the 10 to $30 \mathrm{ps}$ before it relaxed at 80 to 100 ps delay time points, which corroborated well with shorter and longer time points collected at LCLS and APS, respectively $[18,26]$. This early study of PYP using the SPB/SFX instrument demonstrated the feasibility of the pump-probe TR-SFX experiments at high repetition rate X-ray lasers and paved the way toward further improvements in the femtosecond temporal resolution (reviewed in [13]).

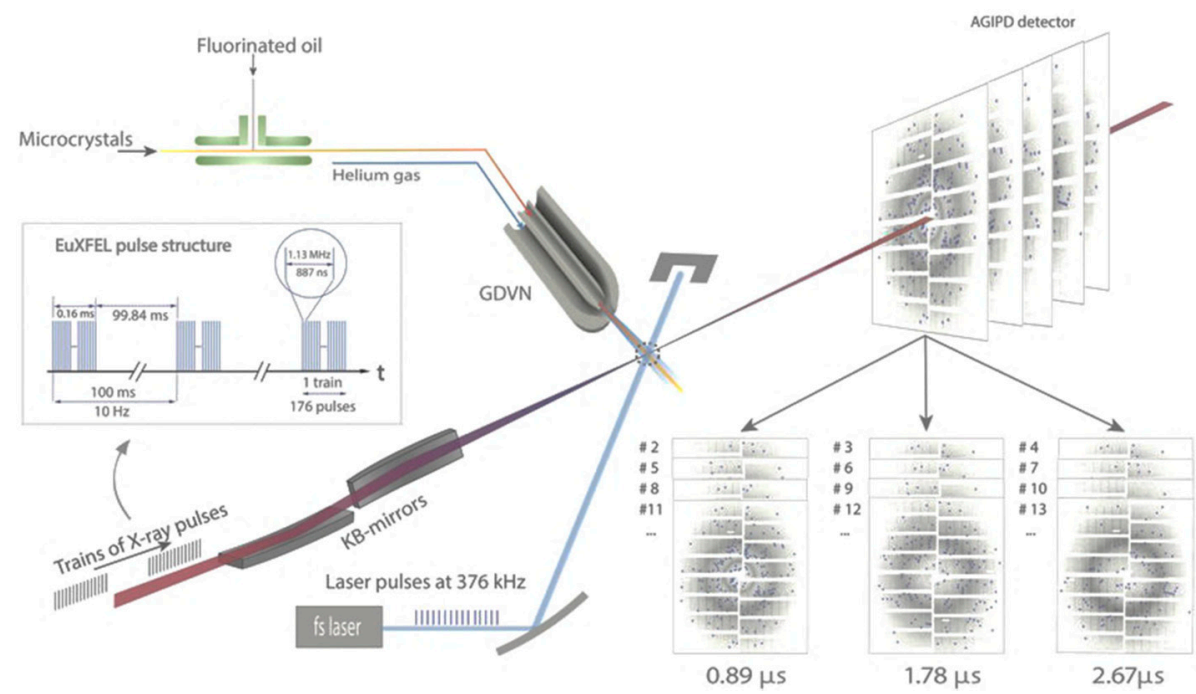

Figure 2. Pump-probe MHz TR-SFX setup using the SPB/SFX instrument of the European XFEL. This setup was implemented during the very first pump-probe TR-SFX experiments [18]. In this setup, X-ray pulses arrive in $1.13 \mathrm{MHz}$ bursts with each burst contains $176 \mathrm{X}$-ray pulses, which repeat every $100 \mathrm{~ms}$. The fs-pump laser delivers $376 \mathrm{kHz}$ pulses in the blue $(\lambda=420 \mathrm{~nm})$, tunable based on the sample requirements, synchronized to the $X$-ray pulses which intersect precisely with the microcrystal jet produced by the GDVN. The pump-probe experimental scheme was designed as follows: one light triggered a $(\Delta t=0.89 \mu \mathrm{s})$ pattern followed by two dark patterns (no light triggering: $(\Delta t=1.78$ and $2.67 \mu \mathrm{s})$ ) to prevent light contamination from the previous laser excitation. The time delay between each pattern equaled $887 \mathrm{~ns}$, corresponding to the X-ray pulse spacing at $1.13 \mathrm{MHz}$ with each pattern representing a different probed crystal. The TR-SFX diffraction patterns were collected on an AGIPD detector (adapted from [18]).

The selection of the optical pump setup, e.g., the laser wavelength, fluence and pulse duration, requires careful experimental planning to achieve as high as possible excited state population while avoiding contamination, which may occur, for instance, due to the multiphoton excitation of the chromophores $[15,58,61,63]$. Such multiphoton excitation can lead to the propagation of radical intermediates, which obscures the biological interpretation of the TR-SFX data [63,64]. Efficient photoexcitation protocol guided by the protein photochemical properties should be followed to allow for the biologically meaningful single-photon absorption of the biomolecules in crystals to avoid such an effect, i.e., multiphoton excitation. Moreover, examining the samples in their crystalline and solution forms with a static and time-resolved optical spectroscopy can significantly assist in the designing of the pump-probe TR-SFX experiments [28,58,61]. Such spectroscopic scanning, e.g., time-resolved UV-visible (transient) spectroscopy, can provide essential information that could help to establish the reaction photocycle, especially when examining a novel system [65], and estimate the time range of each of the photo-intermediates and highlight the differences, if any, between the crystalline and solution forms. As a result, the optimal photoexcitation wavelength and the duration of the photo-intermediates can be determined prior to the pump-probe TR-SFX experiment, thereby assisting in the 
experimental design $[61,64,66,67]$. Grünbein et al. reported comprehensive details of the guidelines and parameters that need to be considered for the photo-excitation setup during pump-probe TR-SFX experiments [62]. As an example, to study the light-induced structural changes of photosystem (PSII) upon two flash (2F) excitation, Shen and co-workers used an ATR-FTIR difference spectroscopy with similar pump laser setup to study the system both in solution and crystalline forms. This study allowed them to estimate the population of the S3 intermediate state upon 2F excitation in the microcrystalline form and used such information for their pump-probe TR-SFX experiment at SACLA [28]. On the other hand, it is also necessary to consider testing the sample delivery devices for their photon-resistance properties before using them for pump-probe TR-SFX experiments in cases when the illumination of the samples in the nozzle is required. This can be achieved by the visual inspection of the fabricated devices after illumination with a range of optical laser powers using a similar laser setup to that of the pump-probe TR-SFX approach [44,45].

\subsection{TR-SFX with Light-Unresponsive Biomacromolecules}

TR-SFX requires a triggering method that is faster than the reaction mechanism steps, the homogenous triggering of the sample, i.e., each single crystal, and a high triggering efficiency. The "low-hanging fruit" of protein targets for TR-SFX studies are photoreactive proteins, which account for only $0.5 \%$ of all proteins, whereas the majority are light-unresponsive proteins $[68,69]$. As research interest moves away from just photoactive proteins, demand for alternative triggering methods increases. However, if the research target is simultaneously light-unresponsive and mechanistically fast, methods such as rapid mixing (ms) [32,70,71] and even temperature jumps (ns) [68] become too slow, with light triggering methods remaining at the fastest timescales (up to fs bond breaking timescale [72]). A promising method to artificially implement light sensitivity is the use of photocages. At a first glance, it may seem that the available compounds and research of new generic photocages will quickly fulfill the need for structural biology compatible photocages. However, the requirements for these experiments become restrictive to many available photocages. The solubility, cleavage rate, light absorption profile and extinction coefficient are all intertwined and need to collectively satisfy the requirements of the experiment [36]. Research has been conducted to find or manufacture new photocages, but even these have their limitations and the demand for new photocages still persists.

There are various photocage factors to consider when planning TR-SFX experiments. The cleavage rate of a photocage relates to the speed from photon absorption to cage release, which is required to be no slower than the phenomenon the experiment is trying to observe, as the slowest event will restrain experiments' time resolution [72]. Photocage light absorption profiles, including both the absorption spectrum and the extinction coefficient, need to be compatible with the sample and the setup. If possible, the pump laser wavelength should not be close to the sample's absorption profile, i.e., proteins $280 \mathrm{~nm}$ or buffer, to minimize laser absorption by molecules other than those of the photocage [36]. The higher the extinction coefficient at a given wavelength, the less laser energy is required for efficient photocage light absorbance and hence, the less unwanted sample absorption, and viceversa for lower coefficients. However, if the extinction coefficient is too high, problems can also arise with triggering homogeneity due to low transmission values along the laser path, as most light will be absorbed at the external crystal layers or in its surrounding buffer [72]. The quantum yield is the fraction of photocages that will release the caged compounds per photon absorbed. If the yield is low, higher pump energy or photocage concentration is required, but then again, these values will need to be compatible with the absorption profile.

Other factors are solubility, compound stability and availability, i.e., either commercially available or manufacturable. Considering all previously mentioned factors and experimental requirements, it becomes obvious that a perfect photocage for each experiment is unlikely; however, a compromise between the various variables is achievable. Photocage-protein interaction is also relevant [36]. Regarding the photocage position 
within the crystals, there are two main possibilities: either the photocage interacts with a specific portion of the protein (i.e., active site) or it does not and is instead found dissolved along the solvent crystal channels. If the photocage specifically interacts with the active site, a high quantum yield will have increased relevancy as each interacting caged compound will only have one chance to trigger the reaction. Each active site where the caged compound remains bound due to low quantum yields will directly contribute to reaction triggering heterogeneity. On the other hand, if the photocage is diffusing into the crystal water channels, a time delay of diffusion toward the active site after de-caging is to be expected at the nanosecond scale. Therefore, it is important to test the experimental setup before attempting the time-limited XFEL experiments. To have a good idea of sample and photocage behavior at the beamlines, a good characterization of the following factors is useful: absorption profile, quantum yield and cleavage rate. After verifying the experimental feasibility and the good compromise of the various variables, including pump laser energy, sample laser absorption and de-caging efficiency, it is also useful to test the delivery method in-house.

An example of photocage usage for TR-SFX experiments is caged nitric oxide (NO) (Figure 3) [34]. Here, the cleavage rates of under tens of microseconds were observed, even though the crystal structure for that particular study slowed the reaction significantly. The photocage quantum yield was 1.4 (each cage released $2 \mathrm{NO}$ ) and the setup had the pump beam split into two sample impinging beams that were $160^{\circ}$ apart for a higher triggering homogeneity. Photocaged soaked crystals were pumped by $6 \mathrm{~ns}$ pulses of $308 \mathrm{~nm}$ wavelength and $0.31-0.51 \mathrm{~mJ} / \mathrm{cm}^{2}$ energy, achieving a compromise between damage (threshold energy of $60 \%$ ) and triggering efficiency as only $50 \%$ of the sites showed NO occupation. Quality dark frames (or time 0 ) together with various other experiments validated the structural information that was obtained [34,73].

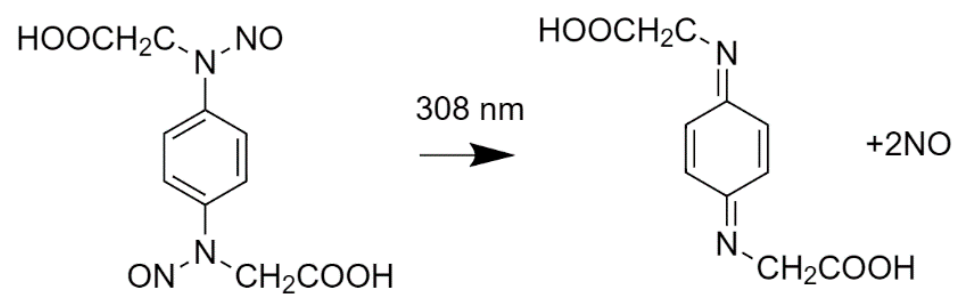

Figure 3. The photocage molecule used in [33]. On the left is the photocaged NO and the corresponding de-caged molecules after laser photoexcitation at $308 \mathrm{~nm}$ are on the right [74].

In conclusion, although photocages enable a method that can provide the fastest timescale triggering of a system (similar accessible timescales up to fs are allowed by the pump-probe method), there is no "one size fits all" photocage because different molecules have different combinations of characteristics, meaning that the best overall cage must be found for each study case. It should be noted that the lack of viable photocages can limit the scope of potential TR-SFX studies. However, for each new uncovered or manufactured photocage molecule that is compatible with the field, the TR-SFX toolbox of photocages becomes larger and more effective.

\section{Outlook}

High repetition rate XFELs offer the unique possibility to collect a very large amount of data in a short period of experimental time; at EuXFEL, it is at least 30 times faster than at other facilities, making it possible to collect a large number of time delays through TR-SFX in a single experiment. This unique feature comes with some constraints, including the injection method, which at present requires a significant number of samples and vacuum operation. In addition, the inherent constraints of TR-SFX, such as the reaction triggering method, need to be addressed.

One of the major limitations preventing new user groups from performing experiments at high repetition rate XFEL sources is sample consumption, although this should be 
overcome when innovative liquid jet delivery methods, such as those described in this review (i.e., co-flow, segmented flow or optical bunching types of injection), are more widely available. For the moment, some challenges remain before they become standard methods, such as the challenge of synchronization with X-ray pulses.

Laser triggering remains the method of choice for the highest time-resolved SFX, as it makes the best use of the short X-ray pulses from the XFELs. The laser parameters in pumpprobe experiments should be defined with care to avoid sample alteration by the laser itself. With the rise of the availability of caged compounds and their use in pump-probe experiments, fast time-resolved studies are becoming possible for catalytic systems that are not inherently light-responsive, thereby opening a variety of new possible experiments.

The preparation and design of a TR-SFX experiment is challenging as it requires the selection of the most relevant delivery method as well as the best suited reaction trigger, whilst considering all limitations and advantages. With all of the current scientific effort in developing and broadening the use of TR-SFX, high repetition rate XFEL sources have a bright future in terms of availability to user groups as well as potential biological targets.

Author Contributions: Conceptualization, R.d.W., D.V.M.M. and F.H.M.K.; writing-original draft preparation, R.d.W., D.V.M.M. and F.H.M.K.; writing-review and editing, R.d.W., D.V.M.M., F.H.M.K. and A.P.M. All authors have read and agreed to the published version of the manuscript.

Funding: We acknowledge the European XFEL and SPB/SFX instrument group for their support.

Institutional Review Board Statement: Not applicable.

Informed Consent Statement: Not applicable.

Data Availability Statement: Not applicable.

Acknowledgments: We acknowledge all staff members of the SPB/SFX instrument at the European XFEL. In particular, we would like to thank Tokushi Sato and Romain Letrun for their critical reading and insightful suggestions for this perspective article.

Conflicts of Interest: The authors declare no conflict of interest.

\section{References}

1. Kendrew, J.C.; Bodo, G.; Dintzis, H.M.; Parrish, R.G.; Wyckoff, H.; Phillips, D.C. A three-dimensional model of the myoglobin molecule obtained by X-ray analysis. Nature 1958, 181, 662-666. [CrossRef] [PubMed]

2. Perutz, M.F.; Rossmann, M.G.; Cullis, A.F.; Muirhead, H.; Will, G.; North, A.C.T. Structure of Hæmoglobin: A three-dimensional fourier synthesis at 5.5-. resolution, obtained by X-ray analysis. Nature 1960, 185, 416-422. [CrossRef]

3. Chapman, H.N.; Fromme, P.; Barty, A.; White, T.A.; Kirian, R.A.; Aquila, A.; Hunter, M.S.; Schulz, J.; Deponte, D.P.; Weierstall, U.; et al. Femtosecond X-ray protein nanocrystallography. Nature 2011, 470, 73-78. [CrossRef] [PubMed]

4. Schlichting, I. Serial femtosecond crystallography: The first five years. IUCrJ 2015, 2, 246-255. [CrossRef] [PubMed]

5. Wilson, M.A. Mapping Enzyme Landscapes by Time-Resolved Crystallography with Synchrotron and X-ray Free Electron Laser Light. Annu. Rev. Biophys. 2022, 51. [CrossRef] [PubMed]

6. Barty, A.; Caleman, C.; Aquila, A.; Timneanu, N.; Lomb, L.; White, T.A.; Andreasson, J.; Arnlund, D.; Bajt, S.; Barends, T.R.M.; et al. Self-terminating diffraction gates femtosecond X-ray nanocrystallography measurements. Nat. Photonics 2012, 6, 35-40. [CrossRef]

7. Neutze, R.; Wouts, R.; van der Spoel, D.; Weckert, E.; Hajdu, J. Potential for biomolecular imaging with femtosecond X-ray pulses. Nature 2000, 406, 752-757. [CrossRef]

8. Nass, K.; Gorel, A.; Abdullah, M.M.; Martin, A.V.; Kloos, M.; Marinelli, A.; Aquila, A.; Barends, T.R.M.; Decker, F.-J; Bruce Doak, R.; et al. Structural dynamics in proteins induced by and probed with X-ray free-electron laser pulses. Nat. Commun. 2020, 11, 1814. [CrossRef]

9. Chapman, H.N. X-ray Free-Electron Lasers for the Structure and Dynamics of Macromolecules. Annu. Rev. Biochem. 2019, 88, 35-58. [CrossRef]

10. Tschentscher, T.; Bressler, C.; Grünert, J.; Madsen, A.; Mancuso, A.; Meyer, M.; Scherz, A.; Sinn, H.; Zastrau, U. Photon Beam Transport and Scientific Instruments at the European XFEL. Appl. Sci. 2017, 7, 592. [CrossRef]

11. Mancuso, A.P.; Aquila, A.; Batchelor, L.; Bean, R.J.; Bielecki, J.; Borchers, G.; Doerner, K.; Giewekemeyer, K.; Graceffa, R.; Kelsey, O.D.; et al. The single particles, clusters and biomolecules and serial femtosecond crystallography instrument of the european XFEL: Initial installation. J. Synchrotron Radiat. 2019, 26, 660-676. [CrossRef] 
12. Allahgholi, A.; Becker, J.; Delfs, A.; Dinapoli, R.; Goettlicher, P.; Greiffenberg, D.; Henrich, B.; Hirsemann, H.; Kuhn, M.; Klanner, R.; et al. The Adaptive Gain Integrating Pixel Detector at the European XFEL. J. Synchrotron Radiat. 2019, 26, 74-82. [CrossRef] [PubMed]

13. Mills, G.; Bean, R.; Mancuso, A.P. First Experiments in Structural Biology at the European X-ray Free-Electron Laser. Appl. Sci. 2020, 10, 3642. [CrossRef]

14. Orville, A.M. Recent results in time resolved serial femtosecond crystallography at XFELs. Curr. Opin. Struct. Biol. 2020, 65, 193-208. [CrossRef]

15. Brändén, G.; Neutze, R. Advances and challenges in time-resolved macromolecular crystallography. Science 2021, 373. [CrossRef]

16. Vakili, M.; Bielecki, J.; Knoška, J.; Otte, F.; Han, H.; Kloos, M.; Schubert, R.; Delmas, E.; Mills, G.; de Wijn, R.; et al. 3D printed devices and infrastructure for liquid sample delivery at the European XFEL. J. Synchrotron Radiat. 2022, 29. [CrossRef]

17. Calvey, G.D.; Katz, A.M.; Schaffer, C.B.; Pollack, L. Mixing injector enables time-resolved crystallography with high hit rate at X-ray free electron lasers. Struct. Dyn. 2016, 3, 054301. [CrossRef] [PubMed]

18. Pandey, S.; Bean, R.; Sato, T.; Poudyal, I.; Bielecki, J.; Cruz Villarreal, J.; Yefanov, O.; Mariani, V.; White, T.A.; Kupitz, C.; et al. Time-resolved serial femtosecond crystallography at the European XFEL. Nat. Methods 2020, 17, 73-78. [CrossRef]

19. Olmos, J.L.; Pandey, S.; Martin-Garcia, J.M.; Calvey, G.; Katz, A.; Knoska, J.; Kupitz, C.; Hunter, M.S.; Liang, M.; Oberthuer, D.; et al. Enzyme intermediates captured "on the fly" by mix-and-inject serial crystallography. BMC Biol. 2018, 16, 59. [CrossRef]

20. Kupitz, C.; Olmos, J.L.; Holl, M.; Tremblay, L.; Pande, K.; Pandey, S.; Oberthür, D.; Hunter, M.; Liang, M.; Aquila, A.; et al. Structural enzymology using X-ray free electron lasers. Struct. Dyn. 2017, 4, 044003. [CrossRef] [PubMed]

21. Martin-Garcia, J.M.; Conrad, C.E.; Coe, J.; Roy-Chowdhury, S.; Fromme, P. Review: Serial Femtosecond Crystallography: A Revolution in Structural Biology. Arch. Biochem. Biophys. 2016, 602, 32. [CrossRef] [PubMed]

22. Schmidt, M. Time-Resolved Macromolecular Crystallography at Pulsed X-ray Sources. Int. J. Mol. Sci. 2019, 20, 1401. [CrossRef] [PubMed]

23. Aquila, A.; Hunter, M.S.; Doak, R.B.; Kirian, R.A.; Fromme, P.; White, T.A.; Andreasson, J.; Arnlund, D.; Bajt, S.; Barends, T.R.M.; et al. Time-resolved protein nanocrystallography using an X-ray free-electron laser. Opt. Express 2012, 20, 2706. [CrossRef] [PubMed]

24. Kupitz, C.; Basu, S.; Grotjohann, I.; Fromme, R.; Zatsepin, N.A.; Rendek, K.N.; Hunter, M.S.; Shoeman, R.L.; White, T.A.; Wang, D.; et al. Serial time-resolved crystallography of photosystem II using a femtosecond X-ray laser. Nature 2014, 513, 261-265. [CrossRef] [PubMed]

25. Nango, E.; Royant, A.; Kubo, M.; Nakane, T.; Wickstrand, C.; Kimura, T.; Tanaka, T.; Tono, K.; Song, C.; Tanaka, R.; et al. A three-dimensional movie of structural changes in bacteriorhodopsin. Science 2016, 354, 1552-1557. [CrossRef] [PubMed]

26. Pande, K.; Hutchison, C.D.M.; Groenhof, G.; Aquila, A.; Robinson, J.S.; Tenboer, J.; Basu, S.; Boutet, S.; DePonte, D.P.; Liang, M.; et al. Femtosecond structural dynamics drives the trans/cis isomerization in photoactive yellow protein. Science 2016, 352, 725-729. [CrossRef] [PubMed]

27. Young, I.D.; Ibrahim, M.; Chatterjee, R.; Gul, S.; Fuller, F.D.; Koroidov, S.; Brewster, A.S.; Tran, R.; Alonso-Mori, R.; Kroll, T.; et al. Structure of photosystem II and substrate binding at room temperature. Nature 2016, 540, 453-457. [CrossRef]

28. Suga, M.; Akita, F.; Sugahara, M.; Kubo, M.; Nakajima, Y.; Nakane, T.; Yamashita, K.; Umena, Y.; Nakabayashi, M.; Yamane, T.; et al. Light-induced structural changes and the site of $\mathrm{O}=\mathrm{O}$ bond formation in PSII caught by XFEL. Nature 2017, 543, 131-135. [CrossRef]

29. Kern, J.; Chatterjee, R.; Young, I.D.; Fuller, F.D.; Lassalle, L.; Ibrahim, M.; Gul, S.; Fransson, T.; Brewster, A.S.; Alonso-Mori, R.; et al. Structures of the intermediates of Kok's photosynthetic water oxidation clock. Nature 2018, 563, 421-425. [CrossRef]

30. Suga, M.; Shimada, A.; Akita, F.; Shen, J.-R.; Tosha, T.; Sugimoto, H. Time-resolved studies of metalloproteins using X-ray free electron laser radiation at SACLA. Biochim. Biophys. Acta Gen. Subj. 2020, 1864, 129466. [CrossRef]

31. Ibrahim, M.; Fransson, T.; Chatterjee, R.; Cheah, M.H.; Hussein, R.; Lassalle, L.; Sutherlin, K.D.; Young, I.D.; Fuller, F.D.; Gul, S.; et al. Untangling the sequence of events during the S $2 \rightarrow \mathrm{S} 3$ transition in photosystem II and implications for the water oxidation mechanism. Proc. Natl. Acad. Sci. USA 2020, 117, 12624-12635. [CrossRef] [PubMed]

32. Pandey, S.; Calvey, G.; Katz, A.M.; Malla, T.N.; Koua, F.H.M.; Martin-Garcia, J.M.; Poudyal, I.; Yang, J.H.; Vakili, M.; Yefanov, O.; et al. Observation of substrate diffusion and ligand binding in enzyme crystals using high-repetition-rate mix-and-inject serial crystallography. IUCrJ 2021, 8, 878-895. [CrossRef]

33. Grünbein, M.L.; Gorel, A.; Foucar, L.; Carbajo, S.; Colocho, W.; Gilevich, S.; Hartmann, E.; Hilpert, M.; Hunter, M.; Kloos, M.; et al. Effect of X-ray free-electron laser-induced shockwaves on haemoglobin microcrystals delivered in a liquid jet. Nat. Commun. 2021, 12, 1-11. [CrossRef] [PubMed]

34. Tosha, T.; Nomura, T.; Nishida, T.; Saeki, N.; Okubayashi, K.; Yamagiwa, R.; Sugahara, M.; Nakane, T.; Yamashita, K.; Hirata, K.; et al. Capturing an initial intermediate during the P450nor enzymatic reaction using time-resolved XFEL crystallography and caged-substrate. Nat. Commun. 2017, 8, 1585. [CrossRef]

35. Josts, I.; Niebling, S.; Gao, Y.; Levantino, M.; Tidow, H.; Monteiro, D. Photocage-initiated time-resolved solution X-ray scattering investigation of protein dimerization. IUCrJ 2018, 5, 667-672. [CrossRef]

36. Monteiro, D.C.F.; Amoah, E.; Rogers, C.; Pearson, A.R. Using photocaging for fast time-resolved structural biology studies. Acta Crystallogr. Sect. D Struct. Biol. 2021, 77, 1218-1232. [CrossRef] [PubMed] 
37. DePonte, D.P.; Weierstall, U.; Schmidt, K.; Warner, J.; Starodub, D.; Spence, J.C.H.; Doak, R.B. Gas dynamic virtual nozzle for generation of microscopic droplet streams. J. Phys. D Appl. Phys. 2008, 41, 195505. [CrossRef]

38. Oberthuer, D.; Knoška, J.; Wiedorn, M.O.; Beyerlein, K.R.; Bushnell, D.A.; Kovaleva, E.G.; Heymann, M.; Gumprecht, L.; Kirian, R.A.; Barty, A.; et al. Double-flow focused liquid injector for efficient serial femtosecond crystallography. Sci. Rep. 2017, 7, 44628. [CrossRef] [PubMed]

39. Grünbein, M.L.; Nass Kovacs, G. Sample delivery for serial crystallography at free-electron lasers and synchrotrons. Acta Crystallogr. Sect. D Struct. Biol. 2019, 75, 178-191. [CrossRef]

40. Hejazian, M.; Balaur, E.; Abbey, B. Recent Advances and Future Perspectives on Microfluidic Mix-and-Jet Sample Delivery Devices. Micromachines 2021, 12, 531. [CrossRef] [PubMed]

41. Vakili, M.; Vasireddi, R.; Gwozdz, P.V.; Monteiro, D.C.F.; Heymann, M.; Blick, R.H.; Trebbin, M. Microfluidic polyimide gas dynamic virtual nozzles for serial crystallography. Rev. Sci. Instrum. 2020, 91, 085108. [CrossRef] [PubMed]

42. Nelson, G.; Kirian, R.A.; Weierstall, U.; Zatsepin, N.A.; Faragó, T.; Baumbach, T.; Wilde, F.; Niesler, F.B.P.; Zimmer, B.; Ishigami, I.; et al. Three-dimensional-printed gas dynamic virtual nozzles for X-ray laser sample delivery. Opt. Express 2016, $24,11515$. [CrossRef]

43. Knoška, J.; Adriano, L.; Awel, S.; Beyerlein, K.R.; Yefanov, O.; Oberthuer, D.; Peña Murillo, G.E.; Roth, N.; Sarrou, I.; VillanuevaPerez, P.; et al. Ultracompact 3D microfluidics for time-resolved structural biology. Nat. Commun. 2020, 11, 657. [CrossRef] [PubMed]

44. Doppler, D.; Rabbani, M.T.; Letrun, R.; Villarreal, J.C.; Kim, D.H.; Gandhi, S.; Egatz-Gomez, A.; Sonker, M.; Chen, J.; Koua, F.H.M.; et al. Co-flow injection for serial crystallography at X-ray free-electron lasers. J. Appl. Crystallogr. 2022, 55. [CrossRef]

45. Echelmeier, A.; Cruz Villarreal, J.; Messerschmidt, M.; Kim, D.; Coe, J.D.; Thifault, D.; Botha, S.; Egatz-Gomez, A.; Gandhi, S.; Brehm, G.; et al. Segmented flow generator for serial crystallography at the European X-ray free electron laser. Nat. Commun. 2020, 11, 4511. [CrossRef] [PubMed]

46. Awel, S.; Bohne, S.; Ebrahimifard, R.; Trieu, H.K.; Bajt, S.; Chapman, H.N. Optical bunching of particles in a liquid flow. Opt. Express 2021, 29, 34394. [CrossRef]

47. Roessler, C.G.; Agarwal, R.; Allaire, M.; Alonso-Mori, R.; Andi, B.; Bachega, J.F.R.; Bommer, M.; Brewster, A.S.; Browne, M.C.; Chatterjee, R.; et al. Acoustic Injectors for Drop-On-Demand Serial Femtosecond Crystallography. Structure 2016, 24, 631-640. [CrossRef]

48. Fuller, F.D.; Gul, S.; Chatterjee, R.; Burgie, E.S.; Young, I.D.; Lebrette, H.; Srinivas, V.; Brewster, A.S.; Michels-Clark, T.; Clinger, J.A.; et al. Drop-on-demand sample delivery for studying biocatalysts in action at X-ray free-electron lasers. Nat. Methods 2017, 14, 443-449. [CrossRef]

49. Sugahara, M.; Mizohata, E.; Nango, E.; Suzuki, M.; Tanaka, T.; Masuda, T.; Tanaka, R.; Shimamura, T.; Tanaka, Y.; Suno, C.; et al. Grease matrix as a versatile carrier of proteins for serial crystallography. Nat. Methods 2014, 12, 61-63. [CrossRef]

50. Meents, A.; Wiedorn, M.O.; Srajer, V.; Henning, R.; Sarrou, I.; Bergtholdt, J.; Barthelmess, M.; Reinke, P.Y.A.; Dierksmeyer, D.; Tolstikova, A.; et al. Pink-beam serial crystallography. Nat. Commun. 2017, 8, 1281. [CrossRef]

51. Moffat, K. Time-Resolved Macromolecular Crystallography. Annu. Rev. Biophys. Biophys. Chem. 1989, 18, 309-332. [CrossRef] [PubMed]

52. Liu, W.; Wacker, D.; Gati, C.; Han, G.W.; James, D.; Wang, D.; Nelson, G.; Weierstall, U.; Katritch, V.; Barty, A.; et al. Serial Femtosecond Crystallography of G Protein-Coupled Receptors. Science 2013, 342, 1521-1524. [CrossRef] [PubMed]

53. Stagno, J.R.; Liu, Y.; Bhandari, Y.R.; Conrad, C.E.; Panja, S.; Swain, M.; Fan, L.; Nelson, G.; Li, C.; Wendel, D.R.; et al. Structures of riboswitch RNA reaction states by mix-and-inject XFEL serial crystallography. Nature 2017, 541, 242-246. [CrossRef] [PubMed]

54. Sato, T.; Letrun, R.; Kirkwood, H.J.; Liu, J.; Vagovič, P.; Mills, G.; Kim, Y.; Takem, C.M.S.; Planas, M.; Emons, M.; et al. Femtosecond timing synchronization at megahertz repetition rates for an X-ray free-electron laser. Optica 2020, 7, 716. [CrossRef]

55. Skopintsev, P.; Ehrenberg, D.; Weinert, T.; James, D.; Kar, R.K.; Johnson, P.J.M.; Ozerov, D.; Furrer, A.; Martiel, I.; Dworkowski, F.; et al. Femtosecond-to-millisecond structural changes in a light-driven sodium pump. Nature 2020, 583, 314-318. [CrossRef]

56. Oda, K.; Nomura, T.; Nakane, T.; Yamashita, K.; Inoue, K.; Ito, S.; Vierock, J.; Hirata, K.; Maturana, A.D.; Katayama, K.; et al. Time-resolved serial femtosecond crystallography reveals early structural changes in channelrhodopsin. Elife 2021, 10, e62389. [CrossRef]

57. Yun, J.-H.; Li, X.; Yue, J.; Park, J.-H.; Jin, Z.; Li, C.; Hu, H.; Shi, Y.; Pandey, S.; Carbajo, S.; et al. Early-stage dynamics of chloride ion-pumping rhodopsin revealed by a femtosecond X-ray laser. Proc. Natl. Acad. Sci. USA 2021, 118, e2020486118. [CrossRef]

58. Li, H.; Nakajima, Y.; Nomura, T.; Sugahara, M.; Yonekura, S.; Chan, S.K.; Nakane, T.; Yamane, T.; Umena, Y.; Suzuki, M.; et al. Capturing structural changes of the $\mathrm{S} 1$ to $\mathrm{S} 2$ transition of photosystem II using time-resolved serial femtosecond crystallography. IUCrJ 2021, 8, 431-443. [CrossRef]

59. Palmer, G.; Kellert, M.; Wang, J.; Emons, M.; Wegner, U.; Kane, D.; Pallas, F.; Jezynski, T.; Venkatesan, S.; Rompotis, D.; et al. Pumpprobe laser system at the FXE and SPB/SFX instruments of the European X-ray Free-Electron Laser Facility. J. Synchrotron Radiat. 2019, 26, 328-332. [CrossRef]

60. Kirkwood, H.J.; Letrun, R.; Tanikawa, T.; Liu, J.; Nakatsutsumi, M.; Emons, M.; Jezynski, T.; Palmer, G.; Lederer, M.; Bean, R.; et al. Initial observations of the femtosecond timing jitter at the European XFEL. Opt. Lett. 2019, 44, 1650. [CrossRef] 
61. Grünbein, M.L.; Stricker, M.; Nass Kovacs, G.; Kloos, M.; Doak, R.B.; Shoeman, R.L.; Reinstein, J.; Lecler, S.; Haacke, S.; Schlichting, I. Illumination guidelines for ultrafast pump-probe experiments by serial femtosecond crystallography. Nat. Methods 2020, 17, 681-684. [CrossRef] [PubMed]

62. Pandey, S.; Poudyal, I.; Malla, T.N. Pump-Probe Time-Resolved Serial Femtosecond Crystallography at X-ray Free Electron Lasers. Crystals 2020, 10, 628. [CrossRef]

63. Nass, K. Radiation damage in protein crystallography at X-ray free-electron lasers. Acta Crystallogr. Sect. D Struct. Biol. 2019, 75, 211-218. [CrossRef]

64. Coquelle, N.; Sliwa, M.; Woodhouse, J.; Schirò, G.; Adam, V.; Aquila, A.; Barends, T.R.M.; Boutet, S.; Byrdin, M.; Carbajo, S.; et al Chromophore twisting in the excited state of a photoswitchable fluorescent protein captured by time-resolved serial femtosecond crystallography. Nat. Chem. 2018, 10,31-37. [CrossRef]

65. Inoue, K.; Koua, F.H.M.; Kato, Y.; Abe-Yoshizumi, R.; Kandori, H. Spectroscopic Study of a Light-Driven Chloride Ion Pump from Marine Bacteria. J. Phys. Chem. B 2014, 118, 11190-11199. [CrossRef] [PubMed]

66. Hutchison, C.D.M.; Kaucikas, M.; Tenboer, J.; Kupitz, C.; Moffat, K.; Schmidt, M.; van Thor, J.J. Photocycle populations with femtosecond excitation of crystalline photoactive yellow protein. Chem. Phys. Lett. 2016, 654, 63-71. [CrossRef]

67. Miller, R.J.D.; Paré-Labrosse, O.; Sarracini, A.; Besaw, J.E. Three-dimensional view of ultrafast dynamics in photoexcited bacteriorhodopsin in the multiphoton regime and biological relevance. Nat. Commun. 2020, 11, 1240. [CrossRef]

68. Thompson, M.C.; Barad, B.A.; Wolff, A.M.; Sun Cho, H.; Schotte, F.; Schwarz, D.M.C.; Anfinrud, P.; Fraser, J.S. Temperature-Jump Solution X-ray Scattering Reveals Distinct Motions in a Dynamic Enzyme. Nat. Chem. 2019, 11, 1058. [CrossRef]

69. Kubelka, J. Time-resolved methods in biophysics. 9. Laser temperature-jump methods for investigating biomolecular dynamics. Photochem. Photobiol. Sci. 2009, 8, 499-512. [CrossRef]

70. Mehrabi, P.; Schulz, E.C.; Dsouza, R.; Müller-Werkmeister, H.M.; Tellkamp, F.; Dwayne Miller, R.J.; Pai, E.F. Time-resolved crystallography reveals allosteric communication aligned with molecular breathing. Science 2019, 365, 1167-1170. [CrossRef]

71. Schmidt, M. Reaction Initiation in Enzyme Crystals by Diffusion of Substrate. Crystals 2020, 10, 116. [CrossRef]

72. Levantino, M.; Yorke, B.A.; Monteiro, D.C.F.; Cammarata, M.; Pearson, A.R. Using synchrotrons and XFELs for time-resolved X-ray crystallography and solution scattering experiments on biomolecules. Curr. Opin. Struct. Biol. 2015, 35, 41-48. [CrossRef] [PubMed]

73. Terasaka, E.; Yamada, K.; Wang, P.H.; Hosokawa, K.; Yamagiwa, R.; Matsumoto, K.; Ishii, S.; Mori, T.; Yagi, K.; Sawai, H.; et al. Dynamics of nitric oxide controlled by protein complex in bacterial system. Proc. Natl. Acad. Sci. USA 2017, 114, 9888-9893. [CrossRef] [PubMed]

74. Namiki, S.; Arai, T.; Fujimori, K. High-Performance Caged Nitric Oxide: A New Molecular Design, Synthesis, and Photochemical Reaction. J. Am. Chem. Soc. 1997, 119, 3840-3841. [CrossRef] 